C.I.H. BROCAR N. ${ }^{\circ} 13.1987$ - Págs. 57-63

\title{
EL TRIBUNAL INQUISITORIAL DE LOGROÑO
}

\author{
Beñat ZINSKO GARMENDIA*
}

\begin{abstract}
El autor plantea una aproximación al estudio del Tribunal de Logroño a través de las «relaciones de causas», de las que, con la ayuda de la informática, ha llegado a analizar más de 6.000. El resultado es una cuantificación de los diferentes tipos de delitos perseguidos por el Tribunal: seguidores de la secta de Mahoma, fornicadores, blasfemos, judaizantes, brujos o hechiceros, ordenados en función del número de casos y de su significación porcentual. Como el autor dice, el trabajo es un resumen de sus investigaciones.
\end{abstract}

The author, who formulates an approach to the study of the 'Tribunal de Logroño' by means of the 'relaciones de causas', with the help of computer programming, has analyzed more than 6,000 cases. The result is quantification of the different types of crimes prosecuted by the tribunal: followers of the sect of Mohamet, fornicators, blasphemous, judaic sympathizers, witches, ordered according to the number of cases and their percentage importance. As the author says, the work is a résumé of his investigations.

Hasta estos últimos años, el tribunal inquisitorial de Logroño no había llamado la atención de los historiadores, si exceptuamos los dos excelentes artículos de J. Simón Díaz que iniciaron la investigación sobre este tribunal ${ }^{1}$. Desde entonces, dos investigadores esencialmente han manejado su documentación, con dos problemáticas muy distintas: Iñaki Reguera que estudia la génesis y la primera parte de la historia de dicha institución y Gustav Henningsen, en un libro donde el protagonista es el «abogado de las brujas», el Inquisidor Alonso de Salazar y Frías, el que mejor entendió el fenómeno de la brujería² .

* Universidad de Toulouse - Le Mirail.

1. Simón Díaz, J., «La Inquisición en Logroño, 1570-1580», Berceo, I, (1946), pp. 89-119 y «La Inquisición en Logroño, 1580-1600», Berceo, III, (1948), pp. 83-96.

2. Reguera, I., La Inquisición española en el País Vasco, San Sebastián, Ed. Txertoa, 1984. HeNNINGSEN, G., The Witche's advocate, Nevada, 1980; hay edición en castellano: El abogado de las brujas. Brujería vasca e Inquisición española, Madrid, Alianza editorial, 1983. Se puede señalar también otro trabajo de reciente aparición a partir del material del tribunal de Logroño: Cristóbal MARTín, M-A., «La Inquisición de Logroño: mentalidad popular en el agro riojano», Segundo Coloquio sobre Historia de la Rioja, Logroño, 1986, tomo II, pp. 83-90. 
A pesar del gran valor de ambos estudios, falta mucho por conocer y cabe plantearse el porqué de esta falta de interés:

- ¿Este tribunal, abandonado por los historiadores, es acaso tan común que no ha merecido estudio propio? No lo creo, después de varios años dedicados a manejar sus papeles: al revés, me parece lleno de aspectos hasta sorprendentes y muy dignos de ser profundizados. Hubiera podido merecer el apodo de «tribunal-frontera» o de «tribunal-filtro», por su acción en contra de las ideas nuevas, en defensa de la España profunda, mayor baluarte de la fe católica. Basta leer la correspondencia entre el tribunal y la Suprema, o las mismas relaciones de causas para darse cuenta claramente del papel que desempeña: ante todo hay que eliminar las novedades peligrosas (controlando el cargamento de los barcos, por ejemplo).

- Pero esta misma misión la cumplen también otros tribunales como los de Zaragoza o de Barcelona. ¿Tendrá entonces el de Logroño algo específico que lo distingue de otros tribunales regionales? Creo que su peculiaridad reside en su posición de frontera: frontera exterior, pero también frontera interior ¿no sería su mejor definición la de «tribunal vasco-castellano»? teniendo Logroño la singularidad de abarcar en su distrito a dos comunidades o étnias, con personaiidad propia, muy contrastada, los vascos y los castellanos.

Tenía el tribunal (véase mapa) unos $33.000 \mathrm{~km} .{ }^{2}$ que se repartían casi igualmente entre el País Vasco $\left(17.682 \mathrm{Km}^{2}\right.$, o sea el $53 \%$ ) y los territorios castellanos que abarcaban la Montaña santanderina, parte de la provincia de Burgos (Bureba y Montañas), La Rioja y un pequeño trozo de la de Soria (partido de Agreda).

Después de varias peregrinaciones -Durango, Osma, Durango, Calahorra (iq), Pamplona, Estella (i?), Tudela- la sede del tribunal se fija en Calahorra en 1523, a raíz de la invasión de Navarra por su antiguo soberano, ilegalmente desposeído por el Católico en $1512 \ldots$

Hasta 1560 , los únicos problemas, fuera de la actividad propia del tribunal, residen en el ensanchamiento del distrito ${ }^{3}$. Ya en los años 1540, los inquisidores calagurritanos empiezan a mirar hacia el inmenso dominio del tribunal de Valladolid, sobre todo en dirección de la Montaña y de los Montes de Oca. Finalmente, los inquisidores Temiño e Ybarra verán sus esfuerzos recompensados a principios de los años 1560 , cuando después de una investigación y ante las reclamaciones de los de Calahorra, la Suprema fija de manera definitiva los límites del tribunal vascocastellano.

Pero, rápidamente, otra pugna, más callada, aparece, al pretender Logroño ser sede del tribunal. Se puede seguir la lucha entre los seguidores de ambas ciudades a través de las cartas a la Suprema.

Esta, al mandar en 1569, a d. Gerónimo Manrique, inquisidor de Valencia, además de averiguar si las penas que se habían impuestos a dos oficiales del tribunal se ejecutaban con el rigor debido, le encarga un análisis de la situación y unas conclusiones sobre el problema de la sede definitiva del tribunal. No parece que Manrique haya vacilado mucho: se muestra en su correspondencia partidario entu-

3. Sobre este problema, v. Contreras, J., y Dedieu, J.P., «Geografía de la Inquisición española. La formación de los distritos, 1470-1820», Hispania, XL, n. ${ }^{\circ} 144$ (1980), pp. 37-93. 
siasta de Logroño. No sabemos si influye en su decisión únicamente el pésimo ejemplo que daban los miembros del tribunal o las cargas numerosas que denuncia a lo largo de su visita y que dañan la imagen que quiere dar de sí la Inquisición. Habrá más razones, seguro, pero no aparecen explícitas en la correspondencia.

Sea lo que fuere, el propio Manrique dirige la mudanza y al menos desde principios de julio de 1570 , reside en Logroño el Santo Tribunal. No se sabe la fecha exacta pero sí que el primer auto de fe se celebra el 18 de octubre; podemos añadir que aún están los inquisidores en Calahorra a mediados de mayo. De aquí en adelante, y hasta la abolición de la Inquisición en 1820, la sede del tribunal no se modificará. Al menos, es lo que se suele creer...

Nuestra aproximación al tribunal se hizo esencialmente a través de la lectura de las relaciones de causas. Se trata de resúmenes de pleitos mandados de manera regular por los tribunales locales a la Suprema que después de analizarlos enviaba consejos y/o órdenes a los inquisidores para, en su caso, dictar sentencias más graves o más leves (i!) y que también, podía rectificar puntos de procedimiento.

En una primera fase, analicé 2.350 relaciones de causas cubriendo los años 1582-16354. Después intenté abarcar toda la actividad del tribunal vasco-castellano desde las primeras relaciones conocidas (1537) hasta su sustitución por las alegaciones fiscales en 1700: esto representa una masa enorme de documentación (más de 6.000 causas) que trataremos con la ayuda de la informática.

Cabe puntualizar que estas 6.000 relaciones sólo representan las que he podido localizar y no el conjunto de las causas que pasaron por delante del tribunal. Faltan muchas, que desaparecieron de distintas maneras:

- idestrucción de documentos cuando la ocupación francesa? Es muy probable aunque todavía no he podido encontrar prueba tajante de ello;

- existencia -probada ésta- de un gran desorden en los papeles de la misma Inquisición: cuando encuadernaron en libros, documentos sueltos y legajos, algunos de ellos no se pusieron en su sitio «natural». Y así es que he podido encontrar (después de I. Reguera) una lista de Logroño en los papeles del tribunal de Zaragoza ${ }^{5}$, pero sobre todo documentación de los tribunales de Valencia, Barcelona, Ciudad Rodrigo en los papeles de Logroño....

- normalmente las relaciones de causas se encuentran en los libros 831 a 839, pero es muy frecuente hallar más en los libros de correspondencia con la Suprema;

- tampoco se puede desconocer el riesgo potencial de pérdida y/o de destrucción en las diversas y sucesivas mudanzas de dicha documentación;

- por fin, queda un elemento sin cuantificar iel robo! El ejemplo más claro es la «desaparición» de las conclusiones finales de Manrique donde, justamente, daba un resumen de sus instrucciones al tribunal de Calahorra así como la lista de las cargas que pesaban sobre cada uno de los miembros del tal tribunal. Sólo quedan, de este cuadernillo, en un libro por lo demás muy bien conservado, la primera y la última página.

5. - $\mathrm{d}^{\circ}-$, ibid., libro 988 , fo 237. 
Todas estas razones, y otras ${ }^{6}$, hacen que es casi seguro que con más búsquedas, sería posible encontrar algunos centenares de acusados más y tener una visión aún más completa de la actividad de nuestro tribunal. A modo de ejemplo, sólo decir que en una estancia de quince días en Madrid, pude encontrar a unos 450 acusados desconocidos hasta ahora y, en consecuencia, no incluidos en el «banco de datos» de G. Henningsen que censa el número de acusados juzgados por los distintos tribunales inquisitoriales, de manera que superamos en un 30\%, aproximadamente, la cifra que daba este autor para Logroño7. El historiador danés había localizado, el 1-XII-1977, para 18 de los 21 tribunales inquisitoriales, 42.214 relaciones de causas, de las cuales 4.296 pertenecían al de Logroño, o sea un $10 \%$ del total de los acusados. Esto demuestra una fuerte subrepresentación de nuestro tribunal en relación con la media nacional.

Ahora bien, centrándonos en el período 1585-1635, vamos a estudiar los rasgos principales de los acusados que comparecieron ante los inquisidores en la capital riojana.

Aunque pueda sorprender, los más numerosos son los de la «secta de Mahoma» con 481 acusados, o sea el $20,5 \%$ del total de los que pasaron ante los jueces logroñeses en dicho período (véase cuadro adjunto). Se concentran en el sureste del distrito alrededor de Aguilar de Río Alhama (165), Agreda (157), así como en Torellas (12). Un segundo foco existe en las Montañas de Burgos, pero en un único pueblo, Bustillo (de Villarcayo), cerca de Medina de Pomar, con 81 acusados. Por fin, se pueden encontrar algunos moriscos más en el valle del Ebro, en Haro (21), Logroño (5), Nájera (5).

Se les sospecha (y no sin razón por cierto) de practicar la religión islámica, prohibida en España desde 1521.

Entre 1582 y 1605 los moriscos representan entre 21,5 y 41,5 del total de los acusados, siendo los quinquenios 1586-90 (172 acusados, 40,5\%) y 1601-05 (71, el $41,5 \%)$ los de mayor represión antimorisca. Después de esta fecha, sólo aparecen de manera casual: de 2,3 a $3,5 \%$ de 1606 a 1620 , fecha en que desaparecen por completo.

No hay verdadera diferenciación entre los sexos ya que son 251 las mujeres y 230 los varones. Conocemos el oficio de éstos en el $92 \%$ de los casos; 5 oficios agrupan más del $75 \%$ de ellos: campesinos (26), olleros (30), arrieros (27), zapateros (31) y, por fin, herreros (45).

El segundo delito es el de los «fornicadores» con 366 acusados $(15,70 \%)$; este alto nivel es algo sorprendente. Se trata ante todo de varones, acusados de haber practicado la simple fornicación o hasta sólo de haber hablado de ella. No hay variaciones importantes en el tiempo.

Recordemos que la simple fornicación se define como cópula carnal fuera del matrimonio entre dos personas libres con mutuo consentimiento. La represión de este delito era una consecuencia del concilio tridentino. 


\section{EL TRIBUNAL INQUISITORIAL DE LOGROÑO}

De manera general, era en una discusión cuando se planteaba el problema, explicando el futuro acusado que no era pecado mortal... Se trata en nuestro caso, siempre de hombres o mujeres sexualmente maduros que realizaron la fornicación o sólo hablaron del tema. Los varones son mucho más numerosos que las mujeres y en su mayoría son castellanos (66\%), mientras los vascos sólo representan la tercia parte de los que caen en este delito. Este último carácter está pendiente de averiguación (en el largo período 1537-1700) para saber si es un hecho estructural o sólo una característica coyuntural del período corto estudiado hasta ahora (1582-1635).

Siguen casi igualados dos tipos de delitos bastante parecidos: las proposiciones heréticas, malsonantes, erróneas o temerarias (12,3\%) y las blasfemias (11\%). Muchas veces estos dos delitos con el precedente (fornicación) y un cuarto, la controversia matrimonio/celibato $(5,4 \%)^{8}$ están agrupados en los papeles del tribunal de Logroño bajo el apartado "proposiciones diversas», lo que condujo a G. Henningsen (en su banco de datos) a hacer un conjunto único con estas proposiciones. Este tipo de delito parece típico del distrito logroñés que encabeza claramente los demás tribunales españoles con 1.850 causas, seguido por Toledo (1.535), Granada (1.455) y Llerena con tan sólo 990 causas 9 .

Evidentemente, habría que ponderar porcentualmente para tomar en cuenta no sólo las cifras absolutas sino también las relativas. En Logroño, los delitos de «proposiciones» representan el 44\% del total en el período 1582-1635, casi un acusado de cada dos, lo que parece enorme. En todos estos tipos de delitos, la proporción de varones es aplastante ( $85-90 \%$ de los acusados).

Para terminar, hablaré de tres delitos más:

- los luteranos representan tan sólo el 9,6\% de los acusados, y con un total de 244 personas se puede decir que la religión reformada no entró prácticamente en España, mayormente cuando se sabe que el $82 \%$ de estos luteranos son extranjeros, entre los cuales los franceses forman el $67 \%$ y los ingleses el $4,5 \%$. Se prendió a la mayoría de ellos en el País Vasco (75\%) y casi todos son hombres $(97 \%)$.

- los acusados de brujería y hechicería ${ }^{10}$ forman solamente el $6 \%$ del conjunto de los acusados. Sólo el 6\% porque, como dejó demostrado G. Henningsen, si las «brujas» denunciadas en la ola de brujería de los años 1609-1614, ola que por otra parte coincide con la actuación del juez De Lancre en el Labourd (País Vasco francés), fueron muy numerosas, pocas comparecieron ante el tribunal logroñés, porque el inquisidor/abogado $\mathrm{A}^{\circ}$ de Salazar y Frías solucionó muchos casos durante su visita y las «brujas» ya no fueron hostigadas por parte del Santo Oficio.

- dos palabras, por fin, del primer delito perseguido por el tribunal, el judaismo. Con sólo 113 acusados, ocupa el octavo lugar en el distrito de nuestro tribunal. Esto puede parecer sorprendente y lo es en efecto. El conjunto navarro-alavés ${ }^{11}$ con 52

8. La proposición consiste en decir que el estado matrimonial es superior al celibato eclesiástico.

9. Henningsen, G., art. cit.

10. Aparte del trabajo de Henningsen ya citado, se podrán ver los de IdOATE, F., sobre la hechicería en Navarra y la obra de CARo BAROJA, J., Las brujas y su mundo, Madrid, Alianza editorial, 1973.

11. Este foco no aparece en los dos grandes atlas vascos que hacen referencia a los judaizantes, el de Erein, p. 72 y el Atlas de Navarra, p. 52. 
judaizantes está decimado en los últimos años del siglo XVI; también encontramos a un grupo de portugueses (23), siendo los demás acusados de este delito dispersos en el conjunto territorial del tribunal.

Este resumen apretado de mis trabajos sobre las relaciones de causas del tribunal logroñés habrá demostrado, así lo espero, el interés de su estudio, pero también los límites impuestos por el marco cronológico demasiado corto como el que eligimos. La ampliación a los casi dos siglos que corren desde 1537 hasta 1700 permitirá estudiar los problemas en la larga duración, lo que no puede dejar de ser interesante.

\section{CAUSAS JUZGADAS EN LOGROÑO SEGUN EL TIPO DE DELITO}

$(1582-1635)$

\begin{tabular}{|c|c|c|}
\hline Delitos & N. ${ }^{\circ}$ de casos & Porcentaje \\
\hline Moriscos & 481 & 20,60 \\
\hline Luteranos & 224 & 9,60 \\
\hline Brujería & 140 & 6,00 \\
\hline Judaizantes & 113 & 4,85 \\
\hline «Fornicación» & 366 & 15,67 \\
\hline A propósito de los «Estados» & 125 & 5,35 \\
\hline Bigamia & 85 & 3,65 \\
\hline Solicitantes & 62 & 2,65 \\
\hline Palabras heréticas & 288 & 12,35 \\
\hline Blasfemias & 257 & 11,00 \\
\hline Familiares & 77 & 3,30 \\
\hline Palabras contra el Santo Oficio & 35 & 1,50 \\
\hline Varios & 82 & 3,50 \\
\hline TOTAL & 2.335 & 100,02 \\
\hline
\end{tabular}




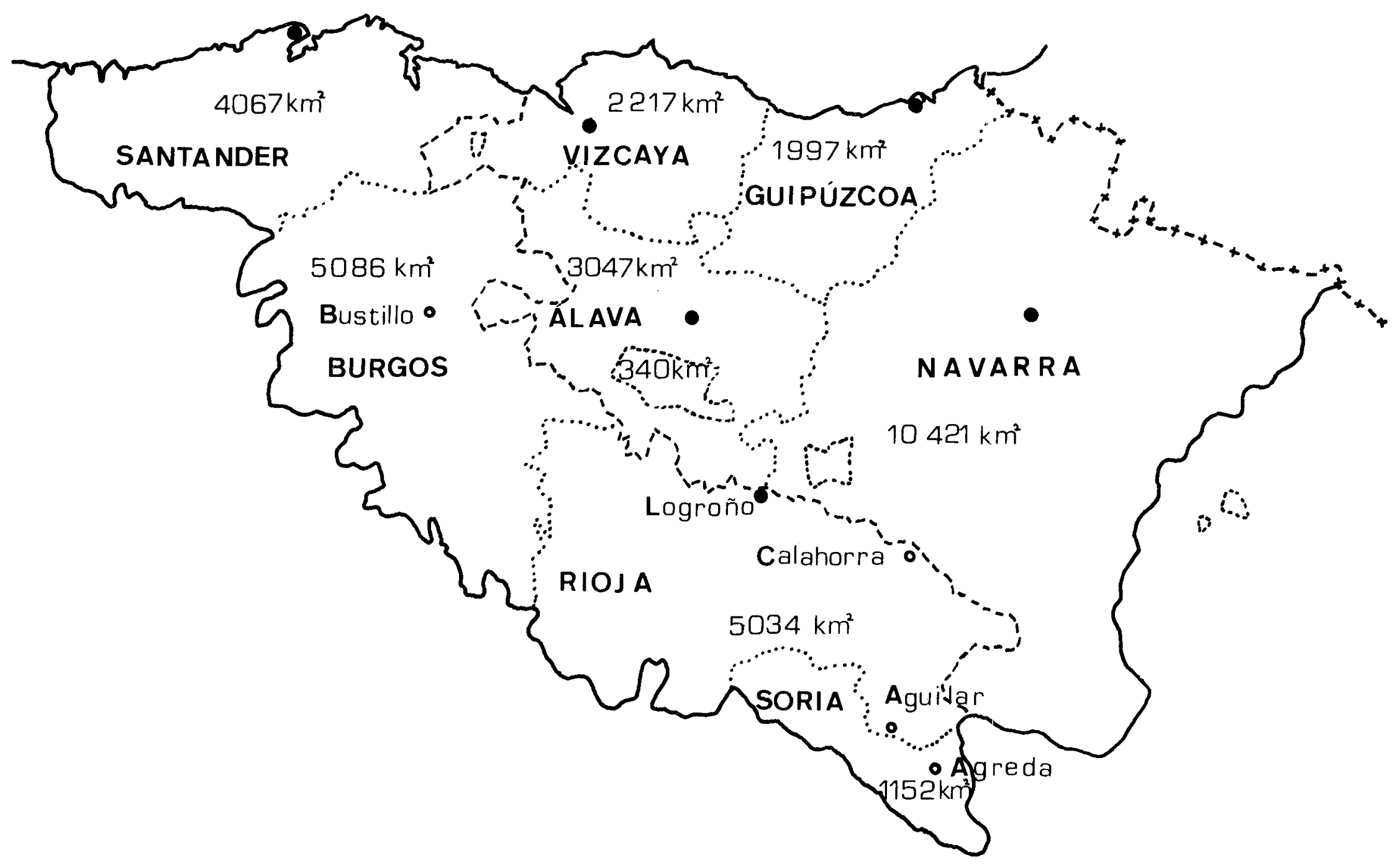


